\title{
On the Formation of Active Regions
}

\author{
Robert F. Stein \\ Department of Physics \& Astronomy, Michigan State University, East Lansing, MI 48824, USA \\ steinepa.msu.edu \\ Åke Nordlund \\ Niels Bohr Institute, University of Copenhagen, DK-2100 Copenhagen, DK \\ aake@nbi.dk
}

\begin{abstract}
Magneto-convection can produce an active region without an initial coherent flux tube. A simulation was performed where uniform, untwisted, horizontal magnetic field of $1 \mathrm{kG}$ strenght was advected into the bottom of a computational domain $48 \mathrm{Mm}$ wide by $20 \mathrm{Mm}$ deep. The up and down convective motions produce a hierarchy of magnetic loops with a wide range of scales, with smaller loops riding "piggy back" in a serpentine fashion on larger loops. When a large loop approaches the surface it produces an small active region with a compact leading spot and more diffuse following spots.
\end{abstract}

Subject headings: Sun; Active Regions; Magnetic Fields; Simulation

\section{Introduction}

The standard paradigm is that active regions form when a coherent flux tube from deep in the convection zone reaches the surface, typically modeled using the thin flux tube approximation (Parker 1955; Fan et al. 1993; Moreno-Insertis et al. 1994; Caligari et al. 1995; Fan 2009; Weber et al.|2011). Cheung et al. (2007); Martínez-Sykora et al. (2008); Weber et al. (2011); Fang et al. (2012) have shown how important the actual convective motions are to the rise of magnetic flux. Recent simulations of active region formation have started from a coherent semi-torus of magnetic field placed in the surface layers of a model solar convection zone (Cheung et al. 2010). Our simulations show that such a coherent structure is not necessary for the formation of an active region. The action of magneto-convection itself produces rising flux tubes, which when they reach the surface can produce an active region.

\section{The Simulation}

We use the STAGGER CODE (Beeck et al.2012) to simulate magneto-convection in a domain $48 \mathrm{Mm}$ wide by $20 \mathrm{Mm}$ deep. This depth is $10 \%$ of the geometric depth of the solar convection zone, but contains 
two thirds of its pressure scale heights. Horizontal directions are periodic and the vertical boundaries are open with plasma and magnetic field moving through them. Because of the large domain dimensions the coriolis force from the solar rotation begins to have some effect, so we include f-plane rotation at a lattitude $30 \mathrm{deg}$ north. The initial state was a snapshot of hydrodyanamic convection which had been relaxed for several turnover times (about 2 days at $20 \mathrm{Mm}$ depth). We then started advecting minimally structured, uniform, untwisted, horizontal magnetic field into the domain by inflows at the bottom. The initial field strength was $200 \mathrm{G}$ (weak enough to have little dynamic effect), which was slowly increased with a 5 hour e-folding time until it reached $1 \mathrm{kG}$ strength and thereafter held constant. The incoming field is at $30 \mathrm{deg}$ to the east-west axis. The active region is not affected by the horizontal boundaries: first, because its size is $25 \mathrm{Mm}$ inside a $48 \mathrm{Mm}$ wide box, second, because it is spreading very slowly with time and, third, because the top boundary is close by (at the temperature minimum) so the magnetic field does not have room to spread out much in the atmosphere and interact with the mirror active regions produced by the horizontal periodicity.

\section{Formation of an active region}
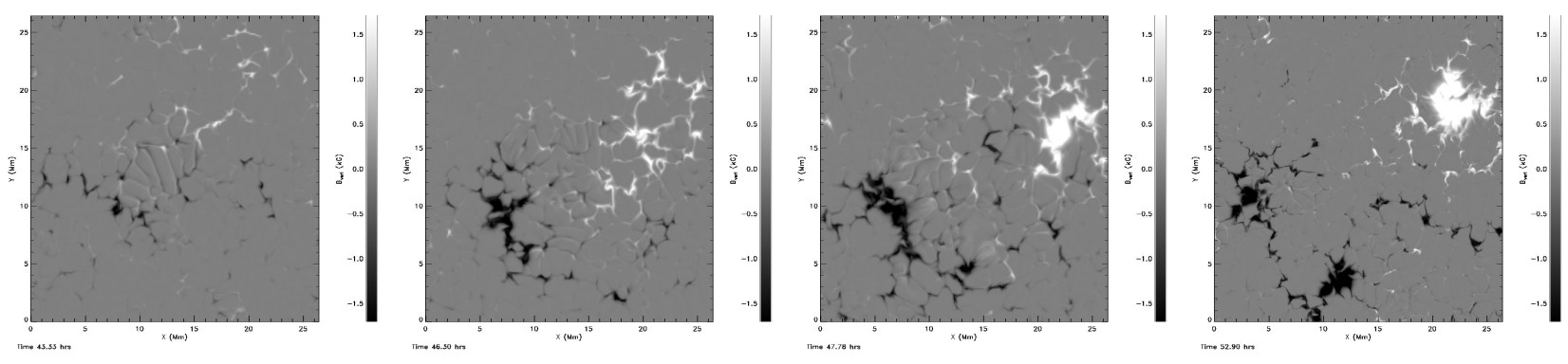

Fig. 1.- Vertical magnetic field. The images are clipped at $\pm 1.7 \mathrm{kG}$, but the actual range is $\pm 3 \mathrm{kG}$. The $\mathrm{x}$-axis is the East-West axis. The field entered in the upflows at the bottom at an angle $30^{\circ}$ to the $\mathrm{x}$-axis. The AR approximately maintains this orientation. The separation of the pores is approximately the size of the supergranule scale convective cells near the bottom of the domain.

As the magnetic field enters the computational domain, convective upflows immeadiately begin to drag portions toward the surface, while convective downflows immeadiately pull other portions down, thus creating magnetic loops. Magnetic field begins to reach the surface after about 20 hours. Smaller scale convective patterns nearer the surface produce smaller scale crenulations in the magnetic loops, giving them a serpentine structure with small loops riding "piggy-back" on larger ones. The field first emerges as small bipoles with mixed polarity over a confined region of $25 \mathrm{Mm}$ square. At about 40 hours, a large flux bundle begins to emerge with rate of $2-4 \times 10^{20} \mathrm{Mx} / \mathrm{hr}$ (Figure 1). The area of major flux emergence is confined by the convective up- and down-flows and does not fill the entire domain. Flux emergence in the remainder of the domain is much smaller. The field first emerges horizontally over granules and then vertical field appears in the intergranule lanes at the ends of the horizontal field. The vertical legs of the bipoles quickly separate. Initially, granules become elongated transverse to the magnetic field. In later emergence events the granules 
are elongated parallel to the horizontal field (Figures 2 and 3). Opposite polarity field migrates to unipolar clusters, sometimes colliding and cancelling in the process (Figure 11). The horizontal components of the emerging field pass through the upper boundary, leaving behind their nearly vertical legs. Individual flux concentrations collect. The leading spot is more compact and rotating and the follow spots more diffuse and non-rotating (Figure 1). The unsigned vertical flux in the active region is a little more than $10^{21} \mathrm{Mx}$ and a similar amount is in the remainder of the domain. This active region has not formed penumbra. As found by Rempel (2012) and upper boundary condition forcing a more horizontal field at the surface is needed to form penumbra. The emerging flux and active region here covers a region of supergranule scale, similar to the sizes of the convective cells at the bottom of the simulation domain. The orientation of the active region is approximately the $30 \mathrm{deg}$ orientation of inflow field at the bottom. The magnetic field lines that emerge are an undulation on a larger, subsurface magnetic structure spanning most of the domain. Eventually, the rate of new flux emergence slows and the space between the spots has little horizontal field (Figure 3 ).
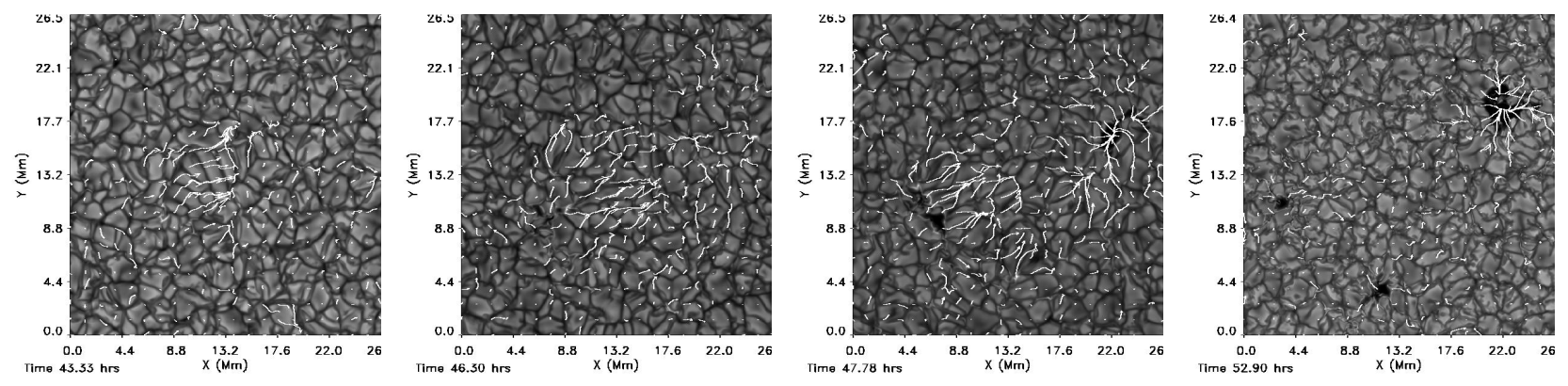

Fig. 2.- Continuum intensity image with horizontal magnetic field vectors superimposed. The images are clipped at $2.3>I /\langle I\rangle>0.5$. The actual range is [0.2,2.5]. In the initial emergence the granules are elongated transverse to the honrizontal field. Thereafter the granules appear elongated along the magnetic field direction.
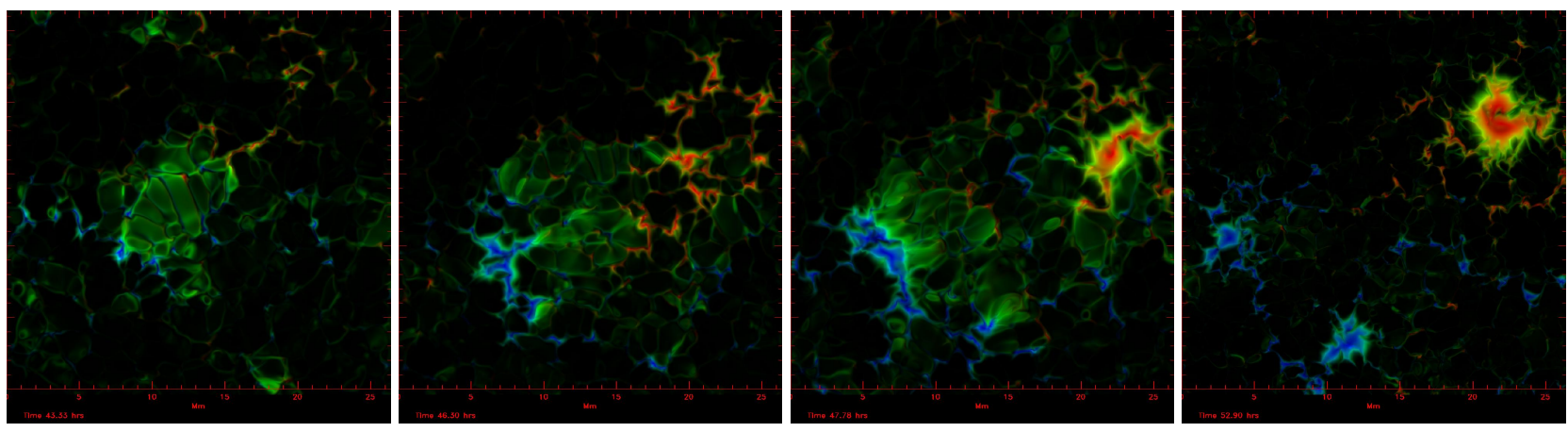

Fig. 3.- Image of horizontal (green) and vertical (blue and red) magnetic field. Magnetic field first emerges horizontally over granules followed by the appearance of vertical field at the granule edges. The orizontal field is quickly swept into the intergranular lanes. 


\section{Summary and Conclusions}

These simulations show that magneto-convection itself can produce the flux tubes that give rise to active regions. The action of up and downflows on an initial supergranule size patch of horizontal field can keep the major portion of the magnetic flux confined to emerge at the surface in a similar supergranule size region. Interesting work for the future will be to investigate the evolution of larger scale magnetic structures, such as those in the flux emergence simulations of Abbett et al. (2000) and Fan (2008), as they pass through the upper convection zone.

Movies of the active region formation are available on http:steinr.pa.msu.edu/ bob/research.html\#AR.

The calculations were performed on the Pleiades cluster of the NASA Advanced Supercomputing Division. RFS is supported by NASA grants NNX12AH49G and NNX08AH44G and NSF grant AGS-1141921. This support is greatly appreciated.

\section{REFERENCES}

Abbett, W. P., Fisher, G. H., Fan, Y. 2000, Astrophys. J, 540, 548

Beeck, B., Collet, R., Steffen, M., Asplund, M., Cameron, R. H., Freytag, B., Hayek, W., Ludwig, H.-G., Schüssler, M. 2012, A\&A, 539, A121

Caligari, P., Moreno-Insertis, F., Schussler, M. 1995, ApJ, 441, 886

Cheung, M. C. M., Rempel, M., Title, A. M., Schüssler, M. 2010, Astrophys. J, 720, 233

Cheung, M. C. M., Schüssler, M., Moreno-Insertis, F. 2007, Astron. Astrophys., 467, 703

Fan, Y. 2008, Astrophys. J, 676, 680

Fan, Y. 2009, Living Reviews in Solar Physics, 6, 1

Fan, Y., Fisher, G. H., Deluca, E. E. 1993, ApJ, 405, 390

Fang, F., Manchester, IV, W., Abbett, W. P., van der Holst, B. 2012, ApJ, 745, 37

Martínez-Sykora, J., Hansteen, V., Carlsson, M. 2008, Astrophys. J, 679, 871

Moreno-Insertis, F., Caligari, P., Schuessler, M. 1994, Sol. Phys., 153, 449

Parker, E. N. 1955, ApJ, 121, 491

Rempel, M. 2012, ApJ, 750, 62

Weber, M. A., Fan, Y., Miesch, M. S. 2011, ApJ, 741, 11 
This preprint was prepared with the AAS LATEX macros v5.2. 